\title{
Investigations on structural, optical and magnetic properties of solution-combustion-synthesized nanocrystalline iron molybdate
}

\author{
KRITHIKADEVI RAMACHANDRAN ${ }^{1}$, SIVA CHIDAMBARAM ${ }^{2}$, BALRAJ BASKARAN ${ }^{1}$, \\ ARULMOZHI MUTHUKUMARASAMY ${ }^{1, *}$ and JOHN BERCHMANS LAWRENCE ${ }^{3}$ \\ ${ }^{1}$ Department of Petrochemical Technology, Anna University - BIT Campus, Thiruchirappalli 620024, India \\ ${ }^{2}$ Department of Physics and Nanotechnology, SRM University, Kattankulathur 603203, India \\ ${ }^{3}$ Department of Electropyro Metallurgy, CSIR-Central Electro-Chemical Research Institute, Karaikudi 630003, India
}

MS received 31 August 2015; accepted 25 May 2016

\begin{abstract}
Iron molybdate $\beta$-Fe $\mathrm{Fe}_{2}\left(\mathrm{MoO}_{4}\right)_{3}$ nanoparticles were synthesized by exploiting the self-propagating hightemperature combustion strategy using hexamine as a fuel. The obtained $\beta$ - $\mathrm{Fe}_{2}\left(\mathrm{MoO}_{4}\right)_{3}$ nanoparticles exhibited the orthorhombic crystalline structure, which is evidenced from the XRD pattern. FT-IR spectrum revealed the existence of stretching and bending vibrations of Mo-O-Mo and $\mathrm{O}-\mathrm{Mo}-\mathrm{O}$ bonding in the nanocrystals. The binding energy peaks in the X-ray photoelectron spectroscopy (XPS) spectrum positioned at 710 and 725,231 and 235 and $530 \mathrm{eV}$, respectively, correspond to the $\mathrm{Fe}(2 \mathrm{p}), \mathrm{Mo}(3 \mathrm{~d})$ and $\mathrm{O}(1 \mathrm{~s})$ orbitals. Absorption spectrum of nanoparticles showed adequate absorbance of visible region photons of the nanoparticles and also optical bandgap value of $\beta-\mathrm{Fe}_{2}\left(\mathrm{MoO}_{4}\right)_{3}$ nanoparticles as $2.26 \mathrm{eV}$, which is calculated using the Kubelka-Munk function. The existence of $\mathrm{Fe}^{3+}$ and $\mathrm{Mo}^{6+}$ in $\beta-\mathrm{Fe}_{2}\left(\mathrm{MoO}_{4}\right)_{3}$ is authenticated with the aid of electron paramagnetic resonance spectrum measurements. The obtained nanoparticles have showed methylene blue dye degradation of $98.4 \%$ under sunlight irradiation.
\end{abstract}

Keywords. Hexamine; iron molybdate; nanocrystalline; solution combustion; photo-degradation.

\section{Introduction}

Binary metal oxide nanomaterials, especially metal molybdates, are fascinating materials nowadays, due to their exhibition of intermingled physiochemical, optical and electrical properties of molybdenum oxide and relevant metal ions [1-5]. The metal molybdates are of $\mathrm{A}-\mathrm{MoO}_{4}$ type binary metal oxide system, in which a divalent metal ion (A) is effortlessly connected to the $\mathrm{MoO}_{4}$ complex. Recently, several metal ions ( $\mathrm{A}=\mathrm{Ni}, \mathrm{Zn}, \mathrm{Fe}, \mathrm{Ca}, \mathrm{Mn}, \mathrm{Mg}, \mathrm{Pb}$ and $\mathrm{Co}$ ) based molybdates had been frequently reported for their synthesis and their characterization portrayal studies [6-10]. These metal molybdates had shown excellence in various fields such as supercapacitors [11], catalysis [12], gas sensors [13] and scintillatormaterials [14]. Such metal molybdates generally exist in two different crystal structures, which are scheelite and wolframite with different cationic radii. The cationic radii are greater than $0.99 \AA$ normally, and the cations form the scheelite structure with Mo occupying the tetrahedral co-ordination and the cations with radii smaller than $0.77 \AA$ form the wolframite structure with Mo occupying the octahedral coordination $[15,16]$. One of the aforementioned metal molybdates, iron molybdate has been pioneering in the application fields such as gas sensing [17],

*Author for correspondence (arulmozhisenthilkumar@gmail.com) methanol-formaldehyde oxidations [18-21], dye degradation [22] and magnetic devices [23]. Iron molybdate nanostructures exhibit diverse morphologies and various preparation methods feasibly produce the iron molybdate nanomaterials, which are not limited to the co-precipitation [24] method, hydrothermal technique [25], solid-state reaction between constituent oxides [26] and high-energy ball milling strategy [27]. The interesting properties of iron molybdate nanoparticles signify the importance of studies on new synthesis routes and their characterization portrayal studies. Here we report the combustion synthesis of iron molybdate nanoparticles obtained from the mixture solution of metal precursors and hexamine. Further, the produced nanoparticles were studied for their structural, optical, magnetic and photocatalytic properties.

\section{Materials and methods}

\subsection{Materials}

Ferric nitrate of Analar grade (99.999\%) was purchased from Fisher Inorganics \& Aromatics Ltd. Sodium molybdate and hexamine were purchased from Otto Chemika, Biochemika reagents. Methylene blue dye was purchased from Fisher Inorganics \& Aromatics Ltd. The chemical reagents were used for experiments without any further purification. 


\subsection{Synthesis of nanocrystalline iron molybdate}

We utilized the self-propagating solution combustion synthesis followed by heat treatment for the iron molybdate nanoparticles formation, where ferric nitrate and sodium molybdate were utilized as precursors. In a typical synthesis, ferric nitrate of $3 \mathrm{wt} \%$ (40.4 g) and sodium molybdate of $3 \mathrm{wt} \%$ (20.59 g) were dissolved with $150 \mathrm{ml}$ of triple-distilled water. Further, $1 \mathrm{wt} \%$ (14.01 g) of hexamine was added to the solution and stirred well until a homogeneous solution was attained. The obtained reaction mixture was transferred to a quartz beaker and then heat treatment was applied to the reaction vessel at $100^{\circ} \mathrm{C}$ using a hot plate arrangement. The reaction mixture was observed for ignition and bursting process during the heat treatment, due to the presence of hexamine. After the completion of bursting process, the resulted particles were sintered at $750^{\circ} \mathrm{C}$ for $4 \mathrm{~h}$ in air using an electric arc furnace. Further, the obtained particles were dissolved well in the solution mixture of water/n-octylamine of volume ratio $1: 1$ in order to dissolve the iron oxide particles, which could also be formed along with the iron molybdate crystals [28]. After $1 \mathrm{~h}$ the powders were collected back from the medium and dried at ambient conditions. The dried particles were used for further characterization. Remarkably the nanoparticles resulted from this synthetic strategy are about $10-11 \mathrm{~g}$, indicating the feasibility of gram level synthesis of nanoparticles.

\subsection{Characterization methods}

The synthesized nanoparticles were characterized by X-ray diffraction technique using a Philips analytical X-ray Diffractometer with $\mathrm{Cu}-\mathrm{K} \alpha$ radiation $(\lambda=1.5406 \AA)$. The FT-IR spectrum of the synthesized nanoparticles was recorded in the range of $400-4000 \mathrm{~cm}^{-1}$ using a PerkinElmer, UK, Paragon-500 model spectrophotometer. The UVvisible absorption spectrum of the nanomaterial was obtained from the spectrophotometer of JASCO model 7800. The $\mathrm{X}$-ray photoelectron spectroscopy (XPS) analysis was performed with a MULTILAB 2000 Base system model with X-ray, Auger and ISS attachments. The electron paramagnetic resonance (EPR) measurements were recorded at microwave frequency $9.857403 \mathrm{GHz}$ with fields corresponding to about $\sim 6500.000 \mathrm{G}$ sweep width using a Bruker Bio Spin Gmbh EPR spectrometer. The size of the synthesized nanoparticles is measured using a transmission electron microscope (TEM) of TECHNAI T20 model.

\subsection{Photocatalytic experiment}

Photocatalytic activity of $\beta-\mathrm{Fe}_{2}\left(\mathrm{MoO}_{4}\right)_{3}$ nanoparticles was examined in terms of methylene blue dye degradation under solar light irradiation. Initially, $10 \mathrm{mM}$ methylene blue dye was dissolved in $30 \mathrm{ml}$ of double distilled water and placed in a beaker. Further, $0.1 \mathrm{~g}$ of $\beta-\mathrm{Fe}_{2}\left(\mathrm{MoO}_{4}\right)_{3}$ nanoparticles was added to the dye solution. The solution was stirred under direct sunlight illumination during a bright day. Temporal variations of degradation of methylene blue dye in the solution were assessed with UV-visible absorption spectroscopic measurements. The degradation efficiency was calculated using the following equation:

$\%$ Degradation rate $=\left[\left(A_{0}-A\right) / A_{0}\right] \times 100$,

where $A_{0}$ and $A$ are, respectively, initial absorbance and absorbance at any instant of time ' $t$ '.

\section{Results and discussion}

\subsection{Synthesis of materials}

Inducement of combustion wave by self-propagation within the reaction medium is the key source of iron molybdate nanoparticles formation. Upon heating, self-sustained chemical reaction could take place between the oxidizers (ferric nitrate and sodium molybdate) and the fuel (hexamine). The self-sustained chemical reaction transmigrates the exothermic heat from the hexamine fuel and forms the metal complexes by travelling the combustion wave throughout the homogenous reaction mixture. The formation of iron molybdate through the self-propagating combustion wave follows the following chemical reactions:

$$
\begin{aligned}
3 \mathrm{Fe}\left(\mathrm{NO}_{3}\right)_{3} & +3 \mathrm{NaMoO}_{4}+\mathrm{C}_{6} \mathrm{H}_{12} \mathrm{~N}_{4} \rightarrow 3 \mathrm{FeMoO}_{4} \\
& +6 \mathrm{CO}_{2}+6 \mathrm{H}_{2} \mathrm{O}+4 \mathrm{~N}_{2}+3 \mathrm{NO}+3 \mathrm{Na}_{2} \mathrm{O} \\
& +(3 / 4) \mathrm{O}_{2}+\mathrm{N}_{2} \mathrm{O} \\
3 \mathrm{FeMoO}_{4} & +(3 / 4) \mathrm{O}_{2} \rightarrow \mathrm{Fe}_{2}\left(\mathrm{MoO}_{4}\right)_{3}+0.5 \mathrm{Fe}_{2} \mathrm{O}_{3}
\end{aligned}
$$

As in the chemical equations, the reaction mechanism directs the formation of both iron oxide particles and iron molybdate particles. In order to have phase-pure materials of iron molybdates, we have dissolved in the solution of mixture of water and n-octylamine, which resulted in dissolution of $\mathrm{Fe}_{2} \mathrm{O}_{3}$ nanoparticles alone. The separation of iron molybdate from the compound mixture is confirmed from the XRD analysis (see figure 1a) after the purification process.

\subsection{Structural analysis}

The powder X-ray diffraction pattern was obtained for the prepared iron molybdate nanoparticles (see figure 1). The crystalline peaks were indexed and were found to be in good agreement with the standard orthorhombic structure of $\beta-\mathrm{Fe}_{2}\left(\mathrm{MoO}_{4}\right)_{3}$ as per the standard JCPDS card no: 85-2287 [2]. In accordance with the chemical reaction that we have anticipated the $\mathrm{Fe}_{2}\left(\mathrm{MoO}_{4}\right)_{3}$ forms, along with the $\mathrm{Fe}_{2} \mathrm{O}_{3}$, a composite structure. The composite structure was also realized at the stages of materials preparation. The XRD pattern obtained for the prepared sample (see supplementary figure S1) before washing with the solution of n-octylamine 
showed the crystalline peaks of both $\beta-\mathrm{Fe}_{2}\left(\mathrm{MoO}_{4}\right)_{3}$ and $\mathrm{Fe}_{2} \mathrm{O}_{3}$. A number of crystalline peaks in the obtained pattern were in good agreement with the standard orthorhombic $\beta$ - $\mathrm{Fe}_{2}\left(\mathrm{MoO}_{4}\right)_{3}$ JCPDS card no: 85-2287 and the remaining of the peaks matched well with the $\mathrm{Fe}_{2} \mathrm{O}_{3}$ structure of JCPDS card number of 89-2810. The absence of all the crystalline peaks of $\mathrm{Fe}_{2} \mathrm{O}_{3}$ particles in the samples obtained after washing with n-octylamine indicates the formation of pure form of $\beta$ - $\mathrm{Fe}_{2}\left(\mathrm{MoO}_{4}\right)_{3}$ particles. The lattice parameters were estimated for the pure form of $\beta-\mathrm{Fe}_{2}\left(\mathrm{MoO}_{4}\right)_{3}$ as $a=10.8$, $b=9.31$ and $c=9.40$, for the obtained crystal system. Further, the average crystalline size of the material was calculated using the Scherrer formula

$$
D=0.9 \lambda / \beta \cos \theta,
$$

where $D$ is the average crystalline size of the material, $\beta$ the full-width at half-maximum (FWHM) of the crystalline peak of the particular plane, $\theta$ the angle at which the crystal plane diffracts and $\lambda$ the wavelength of the X-ray source used. Thus crystalline size was calculated by the above said Scherrer's equation and found to be $39.84 \mathrm{~nm}$. Further, the dislocation density is calculated using the formula

$$
\delta=1 / D^{2}
$$

and thus determined dislocation density was $6.3003 \times 10^{-4}$.

\subsection{FT-IR studies}

The FT-IR spectrum of nanocrystalline iron molybdate (see figure $1 \mathrm{~b}$ ) was recorded within the wavenumber ranges from 400 to $4000 \mathrm{~cm}^{-1}$ to study the chemical bonds and functional groups present in the synthesized $\beta-\mathrm{Fe}_{2}\left(\mathrm{MoO}_{4}\right)_{3}$. The characteristic band observed at $3293.11 \mathrm{~cm}^{-1}$ can be assigned to the stretching of $\mathrm{O}-\mathrm{H}$ bond, due to the surface hydroxyl groups present in the surface of the nanoparticles. The vibration band located at $2226.38 \mathrm{~cm}^{-1}$ is an evidence for the $\mathrm{C}-\mathrm{H}$ stretching of carbon, which was formed by the carbon residues at the material surfaces that had arisen by the combustion process. The band positioned at $1671.4 \mathrm{~cm}^{-1}$ arises from the asymmetrical stretching of nitro-compounds, which are unavoidable species and could be formed by the burning process of combustion synthesis; this is also documented for the chemical reaction mentioned and the synthesis process. The strong and broad band peaks that were positioned around $850-842 \mathrm{~cm}^{-1}$ can be assigned to the tetrahedral species of Mo in $\mathrm{Fe}_{2}\left(\mathrm{MoO}_{4}\right)_{3}$ [29]. The existence of Mo-O-Mo bond was observed from the vibration band, which pointed in the region of $816-860 \mathrm{~cm}^{-1}$ [30]. The coupling bond between oxygen and molybdenum was confirmed by the vibration band spotted in the region of $824.14 \mathrm{~cm}^{-1}$. Mo-O asymmetrical stretching band was observed around $541.53 \mathrm{~cm}^{-1}$.
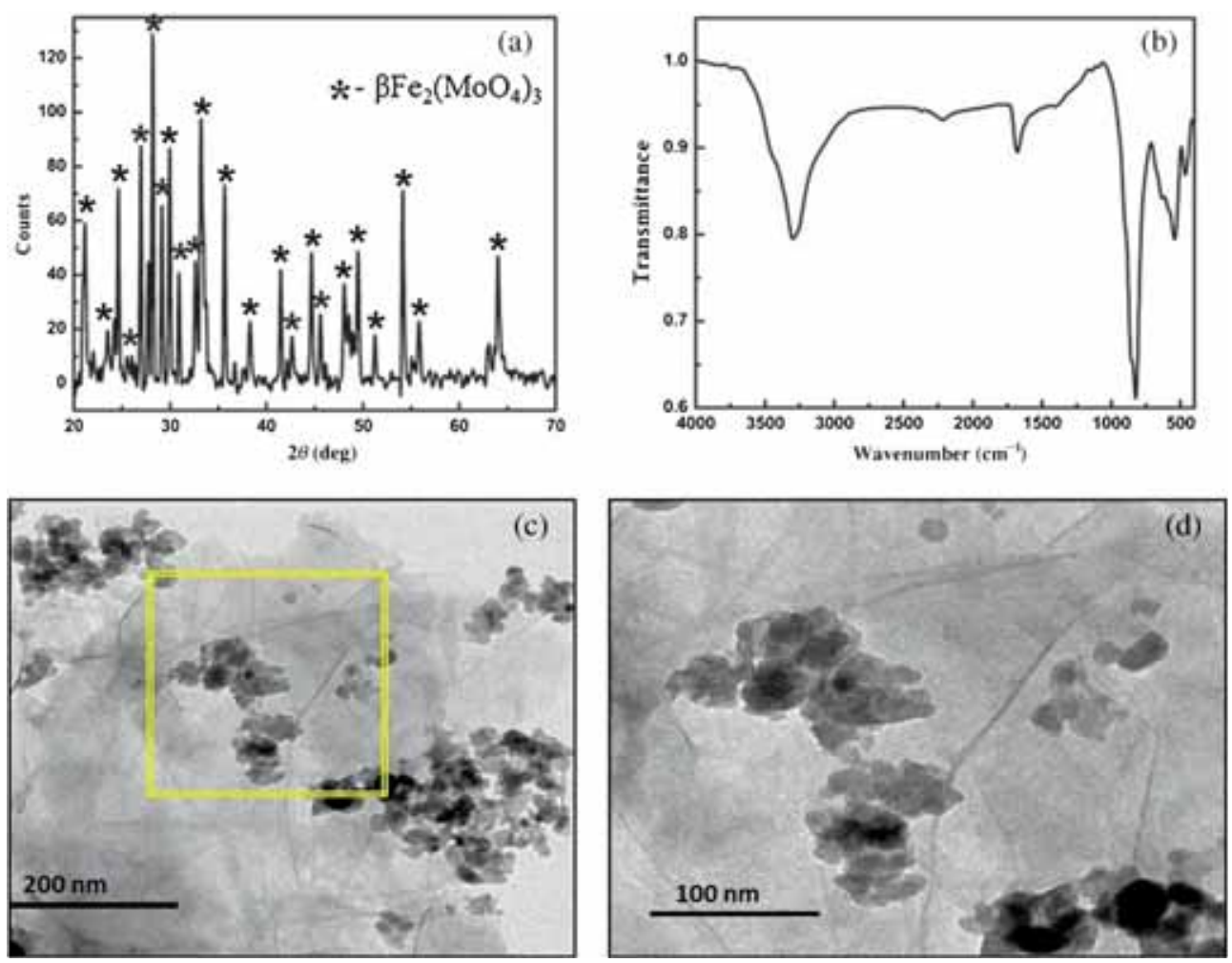

Figure 1. Structural and morphological properties of nanocrystalline iron molybdate nanoparticles: (a) XRD pattern after purification process, (b) FT-IR spectrum, (c) TEM images taken at $200 \mathrm{~nm}$, and (d) TEM images taken at $100 \mathrm{~nm}$ scale. 
The weak band obtained at $456-486 \mathrm{~cm}^{-1}$ can be attributed to Fe-O-Mo stretching vibration mode [29]. The assignment of the vibration bands along with their wavenumber is illustrated in table 1 .

\subsection{Morphological studies}

The morphology of the iron molybdate nanoparticles was examined with TEM analysis as shown in figure 1c and $d$. TEM images clearly indicate particulates of iron molybdate nanoparticles. Also it is evident that the individual particulates aggregated together and form a cluster of nanoparticulates. The particle size majorly falls within 10-40 nm and the particles are non-uniform in structure and size. Due to the lack of use of capping agents in the adopted synthesis procedure, the aggregation is eventually possible and is evident from the TEM images. The magnified image indicates the cluster formation of nano-aggregates.

\subsection{XPS studies}

The XPS studies (see figure 2) exemplify the binding energies of the $\mathrm{Fe}(2 \mathrm{p})$ and $\mathrm{Mo}(3 \mathrm{~d})$ orbitals. The overall survey

Table 1. Assignment of bands and their corresponding wavenumber obtained from FT-IR spectrum.

\begin{tabular}{|c|c|}
\hline $\begin{array}{l}\text { IR band } \\
\text { wavenumber }\left(\mathrm{cm}^{-1}\right)\end{array}$ & Assignment of bands \\
\hline 3293.11 & O-H stretching \\
\hline 2226.38 & $\mathrm{C}-\mathrm{H}$ stretching \\
\hline 1671.4 & Asymmetrical stretching of nitro-compound \\
\hline $850-842$ & Tetrahedral species of $\mathrm{Mo}$ in $\mathrm{Fe}_{2}\left(\mathrm{MoO}_{4}\right)_{3}$ \\
\hline $816-860$ & Mo-O-Mo bond \\
\hline 824.14 & $\begin{array}{l}\text { Coupling bond between oxygen and } \\
\text { molybdenum (O-Mo) }\end{array}$ \\
\hline 635.09 & $\begin{array}{l}\mathrm{MoO}_{3} \text { stretching confirms } \\
\text { the } \mathrm{O}-\mathrm{Mo}-\mathrm{O} \text { bonding }\end{array}$ \\
\hline 541.53 & Mo-O asymmetrical stretching in $\mathrm{MoO}_{4}$ \\
\hline $456-486$ & $\mathrm{Fe}-\mathrm{O}-\mathrm{Mo}$ stretching vibration \\
\hline
\end{tabular}

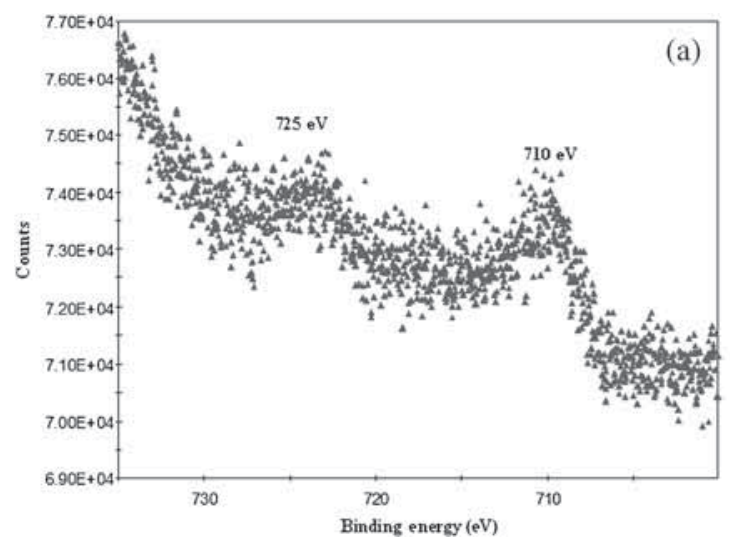

scan spectrum of the synthesized iron molybdate nanoparticles is illustrated (see supplementary figure S2a) with its corresponding binding energies. The binding energy peaks surveyed at the energy positions of 710 and $725 \mathrm{eV}$ (see figure 2a) substantiated the existence of $\mathrm{Fe} 2 \mathrm{P}_{3 / 2}$ for iron III species in $\beta$ - $\mathrm{Fe}_{2}\left(\mathrm{MoO}_{4}\right)_{3}$ and $\mathrm{Fe} 2 \mathrm{P}_{1 / 2}$ in ferrous molybdate, respectively. The binding energy values at 231 and $235 \mathrm{eV}$ (see figure 2b) authenticated the electronic state of molybdenum as $\mathrm{Mo}^{6+}$. Moreover, the lattice site oxygen was examined with the attained binding energy at $530 \mathrm{eV}$ (see supplementary figure S2b). The XPS survey spectrum along with individual scan spectrum provided the valence states of $\mathrm{Fe}$, $\mathrm{Mo}$ and $\mathrm{O}$ in $\beta-\mathrm{Fe}_{2}\left(\mathrm{MoO}_{4}\right)_{3}$ as $+3,+6$ and -2 , respectively. The observed energy positions and the assigned ionic states of the element to the corresponding energy levels are in good agreement with the reported literatures $[31,32]$.

\subsection{Optical properties}

The UV-visible diffuse reflectance spectrum was taken to elucidate the optical properties of the synthesized material. The optical absorption was measured in the UV-visible region and the spectrum is shown as supplementary figure S3a. The spectrum depicted broad absorption in the visible region and narrow absorption in the UV region. The band absorption around $450-500 \mathrm{~nm}$ can be ascribed to the $\mathrm{Fe}-\mathrm{O}$ bonds charge transfer transitions in $\beta-\mathrm{Fe}_{2}\left(\mathrm{MoO}_{4}\right)_{3}$ [33]. The broader absorption suggested the utilization of a larger quantity of visible photons compared with the UV region photons by the prepared $\beta$-Fe $2\left(\mathrm{MoO}_{4}\right)_{3}$ nanoparticles. The band gap value of the nanomaterial was calculated with the aid of the Kubelka-Munk Function. The relationship is expressed as follows:

$$
(K h \gamma)^{0.5}=f(h \gamma)
$$

where $K=(1-R)^{2} / 2 R$ in which $R$ was obtained from the diffuse reflectance data. The inset graph (see supplementary figure S3b) portrays the diffuse reflectance spectrum for

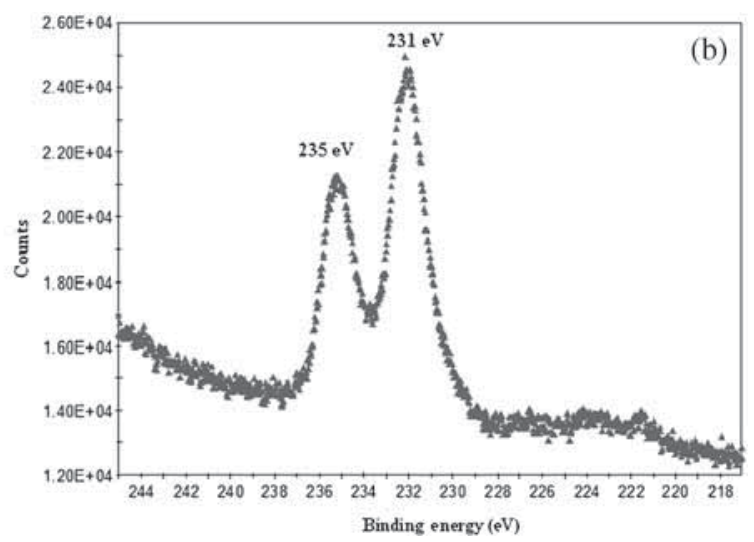

Figure 2. XPS spectrum of iron molybdate nanoparticles: (a) Fe(2p) and (b) Mo(3d) scans. 


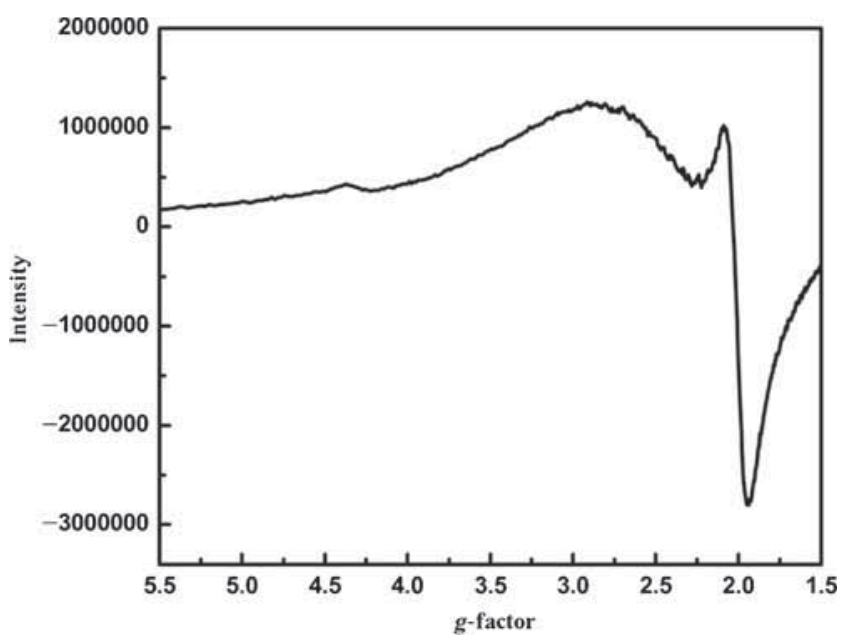

Figure 3. EPR studies of iron molybdate nanoparticles.
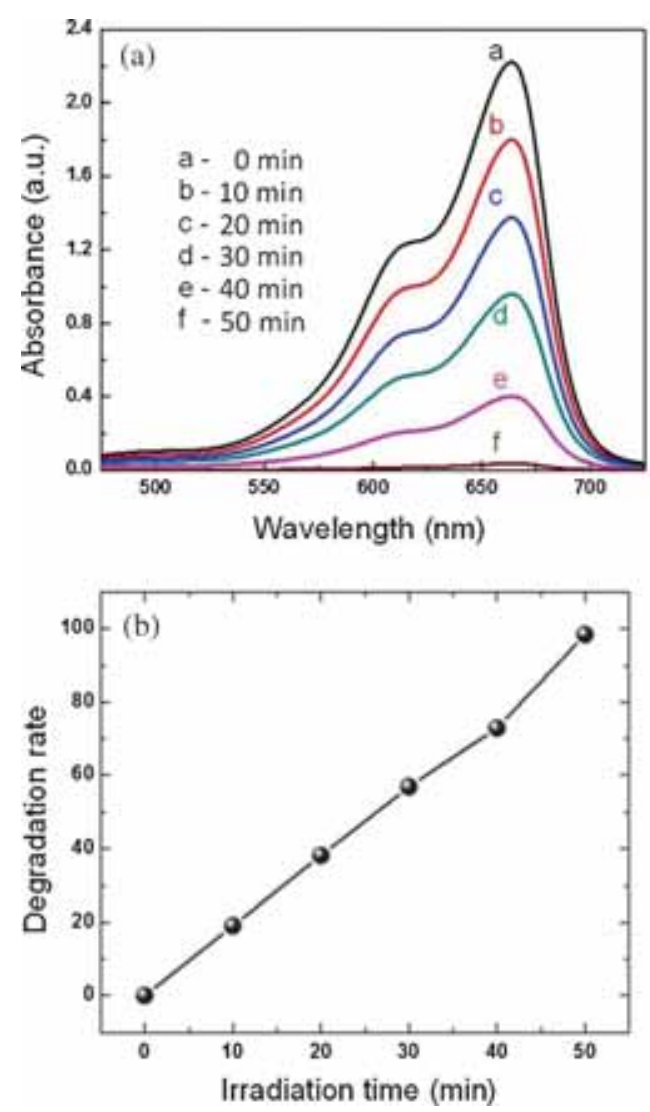

Figure 4. Photocatalytic degradation of methylene blue in the presence of iron molybdate nanoparticles: (a) UV-visible absorption spectrum of methylene blue dye at various time intervals and (b) degradation rate percentage of methylene blue dye molecules at various time intervals.

$\beta-\mathrm{Fe}_{2}\left(\mathrm{MoO}_{4}\right)_{3}$. The optical band gap energy can thus be evaluated from the plot of $(K h \gamma)^{0.5} v s$. the photon energy $(h \gamma)$ as shown in supplementary figure S3b. The intercept of the tangent with the $\mathrm{X}$-axis provided an excellent rough value of $2.26 \mathrm{eV}$ for the synthesized iron molybdate.

\subsection{EPR studies of iron molybdate}

The EPR studies substantiate the magnetic ordering of iron molybdate nanoparticles. The spectrum (see figure 3) explains the EPR studies of iron molybdate particles. For iron molybdate, $\mathrm{Mo}^{5+}$ ions have $g$-factor splitting for lines of $g_{\text {parallel }}=1.95$ and $g_{\text {perpendicular }}=2.02$ as per the Lorentzian line splitting, which is closely in association with the earlier literature reports $[31,34] . \beta-\mathrm{Fe}_{2}\left(\mathrm{MoO}_{4}\right)_{3}$ has 13 peaks, as in the supplementary information, due to the hyperfine splitting and crop-up by the paramagnetic traces of $\mathrm{Fe}^{3+}$ and $\mathrm{Mo}^{5+}$ ions. The observed gyromagnetic factor values are 4.36, 4.21, $2.83,2.61,2.25,2.23,2.02,2,1.95,1.94,1.86,1.76$ and 1.66. Nevertheless, there are common characteristics between the lines of the spectrum, which revealed only 13 instead of 16. Thus it symbolizes the existence of $\mathrm{Fe}^{3+}$ and $\mathrm{Mo}^{6+}$ along with $\mathrm{Mo}^{5+}$.

\subsection{Photocatalytic studies}

The photocatalytic degradation of methylene blue, one of the common organic pollutants, is evaluated for the obtained nanocrystalline iron molybdate particles under direct sunlight. The evolutions of the UV-visible absorption spectra at the wavelength of absorption maximum during the degradation process are demonstrated (see figure $4 \mathrm{a}$ and $\mathrm{b}$ ). Under the illumination of sunlight to the iron molybdate nanoparticles, the observed decrease in the intensity of the characteristic absorption peaks of methylene blue dye indicates the degradation of dye molecule, due to the occurrence of photocatalytic reaction. The establishment of electron-hole pairs under the illumination of light photons on iron molybdate occurs due to the semiconducting nature of the nanocrystalline particles. Thus produced conduction band electrons reduce the surface adsorbed oxygen species $\left(\mathrm{O}_{2}^{-}\right)$. Meanwhile, the holes in conduction band are utilized in the oxidization process for the resulting hydroxyl radicals $\left(\mathrm{HO}^{\circ}\right)$. Thus, a large number of reactive species are present in the iron molybdate nanoparticles, which is evident from the TEM results that facilitated the effective methylene blue dye degradation. Methylene blue molecules of about $98.4 \%$ are able to get degraded within $50 \mathrm{~min}$ in the presence of nanocrystalline iron molybdate.

\section{Conclusion}

Iron molybdate nanoparticles were synthesized using selfpropagating combustion of chemical reagents and thus produced nanoparticles are of substantiating quantity. The sample has been investigated mainly using X-ray diffraction technique, which had shown a fine correlation with the previous reports of orthorhombic structure with associated lattice parameter values. The FT-IR spectrum signified the bonding between the material as $\mathrm{O}-\mathrm{Mo}-\mathrm{O}$ and $\mathrm{Fe}-\mathrm{O}-\mathrm{Mo}$ in $\beta-\mathrm{Fe}_{2}\left(\mathrm{MoO}_{4}\right)_{3}$. The XPS survey spectrum exhibited the valence states of $\mathrm{Fe}, \mathrm{Mo}$ and $\mathrm{O}$ as $+3,+6$ and -2 . The optical properties revealed the band gap energy as $2.26 \mathrm{eV}$ 
from the UV spectrum. The magnetic study by EPR spectroscopy of the material indicates the excellent paramagnetic ordering of the synthesized iron molybdate powders with the ' $g$ ' values indicated by the EPR spectrum. The photocatalytic studies demonstrated the degradation of $98.4 \%$ for methylene blue dye under sunlight irradiation of $50 \mathrm{~min}$. Thus the solution combustion technique proved to be a versatile technique to synthesize nanocrystalline iron molybdate powders, which revolutionizes mainly in all the advanced fields for the applications described.

\section{Electronic supplementary material}

Supplementary material pertaining to this article is available on the Bulletin of Materials Science website (www.ias.ac.in/ matersci).

\section{References}

[1] Sen A and Pramanik P 2001 Mater. Lett. 50287

[2] Ding Y, Yu S H, Liu C and Zang Z A 2007 Chem. Eur. J. 13 746

[3] Changsheng G, Jian X, Shanfeng W, Lei L, Yuan Z and Xiaochen L 2012 J. Cryst. Eng. Comm. 143602

[4] Duan F, Zheng Y and Chen M Q 2011 J. Mater. Lett. 65191

[5] Mikhailik V B, Kraus H, Kapustyanyk V, Panasyuk M, Prots Y, Tsybulskyi V and Vasylechko L J 2008 J. Phys.: Condens. Matter 20365219

[6] Ryu J H, Koo S M, Yoon J W, Lim C S and Shim K B 2006 J. Mater. Lett. 601702

[7] Ahmad G, Dickerson M B, Cherch B C, Cai Y, Jones S E, Naik R R, King J S, Summers C J and Kroger N 2006 J. Adv. Mater. 181759

[8] Madeira L M, Portela M F and Mazzocchi C 2004 J. Catal. Rev. 4653

[9] Vernaleken A, Cohen M G and Metcalf H 2007 J. Appl. Opt. 297117

[10] Wu X, Du J, Li H, Zhang M, Xi B, Fan H, Zhu Y and Qian Y 2007 J. Solid State Chem. 1803288

[11] Bubb D H, Cohen D and Qadri S B 2005 J. Appl. Phys. Lett. 87131909

[12] Madeira L M, Portela M F and Mazzocchia C 2004 J. Catal. Rev. Sci. Eng. 4653
[13] Jacques S D M, Leynaud O, Strusevich D, Beale A M, Sankar G, Martin C M and Barnes P 2006 Int. J. Angew. Chem. 45 445

[14] Abraham Y, Holzwarth N A W and William R T 2000 J. Phys. Rev. B Condens. Matter 621733

[15] Ryu J H, Yoon J W, Lim C S, Oh W C and Shim K B 2005 J. Ceram. Int. 31883

[16] Wu X, Du J, Li H, Zhang M, Xi B, Fan H, Zhu Y and Qian Y 2007 J. Solid State Chem. 1803288

[17] Kersen U and Holappa L 2006 J. Appl. Phys. A 85431

[18] Soares A P V and Portela M F 2004 J. Catal. Rev. 47 125

[19] Brookes C, Wells P P, Cibin G, Dimitratos N, Jones W, Morgan D J and Bowker M 2014 Int. J. ACS Catal. 4243

[20] Soares P V, Portela M F, Kiennemann A, Hilaire L and Millet J M M 2001 J. Appl. Catal. A Gen. 206221

[21] House M P, Cruise N, Holmberg J, Bowker M, Bovin J O and Andersson Soderhjelm A 2008 J. Top. Catal. 50145

[22] Pardehkhorram R, Tajarodi A, Imani M, Ebrahimi S, Koshy P and Sorrell C C 2015 J. Austr. Ceram. Soc. 5194

[23] Sleight A W, Chamberland B L and Weiher J F 1968 J. Inorg. Chem. 71093

[24] Hummadi K K, Hassan K H and Mitchell P C H 2009 J. Eng. Res. 61

[25] Rapposch M H, Anderson J B and Kostiner E 1980 J. Inorg. Chem. 193531

[26] Massarotti V, Flor G and Marini A 1981 J. Appl. Crystallogr. 1464

[27] Radev D D, Blaskov V, Klissurski D, Mitov I and Toneva A 1997 J. Alloys Compd. 256108

[28] Yu R, Li Z, Wang D, Lai X, Xing C and Xing X 2009 J. Solid State Sci. 12056

[29] Singh R N, Madhu Awasthi R and Tiwari S K 2009 Int. J. Hydrogen Energy 344693

[30] Uhlrich J J, Saino J, Lei Y, Edwards D, Davies R, Bowker M, Shaikhutdhinov S and Freund H J 2011 J. Surf. Sci. 605 1500

[31] Soares A P V, Portela M F, Kiennemann A and Hilaire L 2003 J. Chem. Eng. Sci. $\mathbf{5 8} 1315$

[32] Senthilkumar B and Kalaiselvan R 2014 J. Colloids Interface Sci. 426280

[33] Li D, Xue J and Liu M 2015 New J. Chem. 391910

[34] Shenouda A Y 2006 J. Electrochem. Acta 515973 